Correspondence

\title{
Can multiple previous treatment-requiring rejections affect biventricular myocardial function in heart transplant recipients? A two-dimensional speckle-tracking study
}

\author{
Giuseppe Romano a , Giuseppe Maria Raffa ${ }^{\mathrm{b}, *}$, Pamela Licata ${ }^{\mathrm{a}}$, Fabio Tuzzolino ${ }^{\mathrm{c}}$, Cesar Hernandez Baravoglia ${ }^{\mathrm{b}}$, \\ Sergio Sciacca ${ }^{\mathrm{b}}$, Cesare Scardulla ${ }^{\mathrm{a}}$, Michele Pilato ${ }^{\mathrm{b}}$, Patrizio Lancellotti ${ }^{\mathrm{d}}$, Francesco Clemenza ${ }^{\mathrm{a}}$, Diego Bellavia ${ }^{\mathrm{a}}$

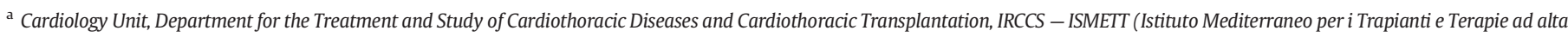 \\ Specializzazione), Via Tricomi 5, 90127 Palermo, Italy

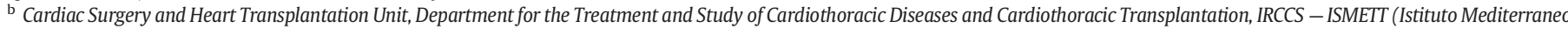 \\ per $i$ Trapianti e Terapie ad alta Specializzazione), Via Tricomi 5, 90127 Palermo, Italy \\ ' Research Office, IRCCS - ISMETT (Istituto Mediterraneo per i Trapianti e Terapie ad alta Specializzazione), Via Tricomi 5, 90127 Palermo, Italy \\ d University of Liège Hospital, GIGA Cardiovascular Sciences, Department of Cardiology, Heart Valve Clinic, CHU Sart Tilman, Liège, Belgium
}

\section{A R T I C L E I N F O}

Article history:

Received 15 January 2016

Accepted 1 February 2016

Available online 3 February 2016

\section{Keywords:}

Echocardiography

Strain

Speckle-Tracking

Heart transplant

Cellular rejection

\section{Introduction}

Echocardiography is the first line imaging modality to assess heart transplantation (HTx) patients, providing accurate informations about graft anatomy and function however, it is not recommended as an alternative to serial endomyocardial biopsy (EMB) in rejection episodes monitoring [1]. Global longitudinal systolic strain (GLS), a measurement of myocardial deformation [1], has been shown sensitive in detecting acute cellular rejection in these patients [2].

It is unclear whether previous episodes of cellular rejection requiring treatment (PreCR), that is a rejection level worse than 1R class [3], could also affect myocardial deformation detected afterwards. The present study aims at examining the role of left (LV) and right ventricular (RV) systolic GLS as a tool to detect subclinical myocardial impairment due to injury-related PreCR in asymptomatic HTx recipients.

\footnotetext{
All authors take responsibility for all aspects of the reliability and freedom from bias of the data presented and their discussed interpretation.

* Corresponding author.

E-mail address: giuseppe.raffa78@gmail.com (G.M. Raffa).
}

\section{Methods}

The study was approved by the IRRB of the "Istituto Mediterraneo per i Trapianti e Terapie ad Alta Specializzazione", IRCCS-ISMETT). Twenty-seven consecutive HTx recipients underwent EMB, from January 1st 2014 through December 1st, 2014, as part of the rejection monitoring protocol, in place at our institute. Patients with signs of coronary allograph vasculopathy $(\mathrm{N}=2)$ diagnosed by medical history or stress test, and those with sub-optimal ultrasound quality $(\mathrm{N}=1)$ were excluded. Contingent acute cellular rejection $>1 \mathrm{R}$ episode at enrollment was considered a confounding factor [2], so that these patients $(\mathrm{N}=3)$ were excluded as well. Finally, 21 patients consented to participate to the study; this group of interest was compared to a sample ( $n=21$ ) of healthy subjects (control group) who were age-, gender-, and ejection fraction (EF)-matched.

The online supplementary file (designed as " $\mathrm{S}$ ") summarizes the study population characteristics, the echocardiographic measurements that have been collected, the statistical methods and the study limitations.

\section{Results}

Mean age of HTx patients was $48 \pm 12$ years, 90\% (19/21) of whom were male; $47 \%$ (10/21) had post-ischemic dilated cardiomyopathy at baseline, 33\% (7/21) idiopathic dilated cardiomyopathy. Arterial hypertension was detected in 61\% (13/21), while 52\% (11/21) suffered from type II diabetes. The average donor age was $30 \pm 12$ years. On average, patients were assessed $41 \pm 61$ months after HTx. Patients' characteristics and number of PreCR $>1 \mathrm{R}$ are shown in Table 1S. Results of the echocardiography and strain analyses in both HTx patients and healthy subjects are reported in Table 1 . Associations between different variables (number of PreCR, standard echocardiography measures, ischemia time, time after HTx and LV-/RV-GLS) are listed in Table 2. Both LVGLS and RVGLS were correlated with the number of episodes of PreCR ( $\mathrm{p}=0.04$ and $\mathrm{p}=0.02$, respectively) (Table 2; Figs. $1 \mathrm{~S}$ and $2 \mathrm{~S})$. The tissue fibrosis was quantified histopathologically according to 

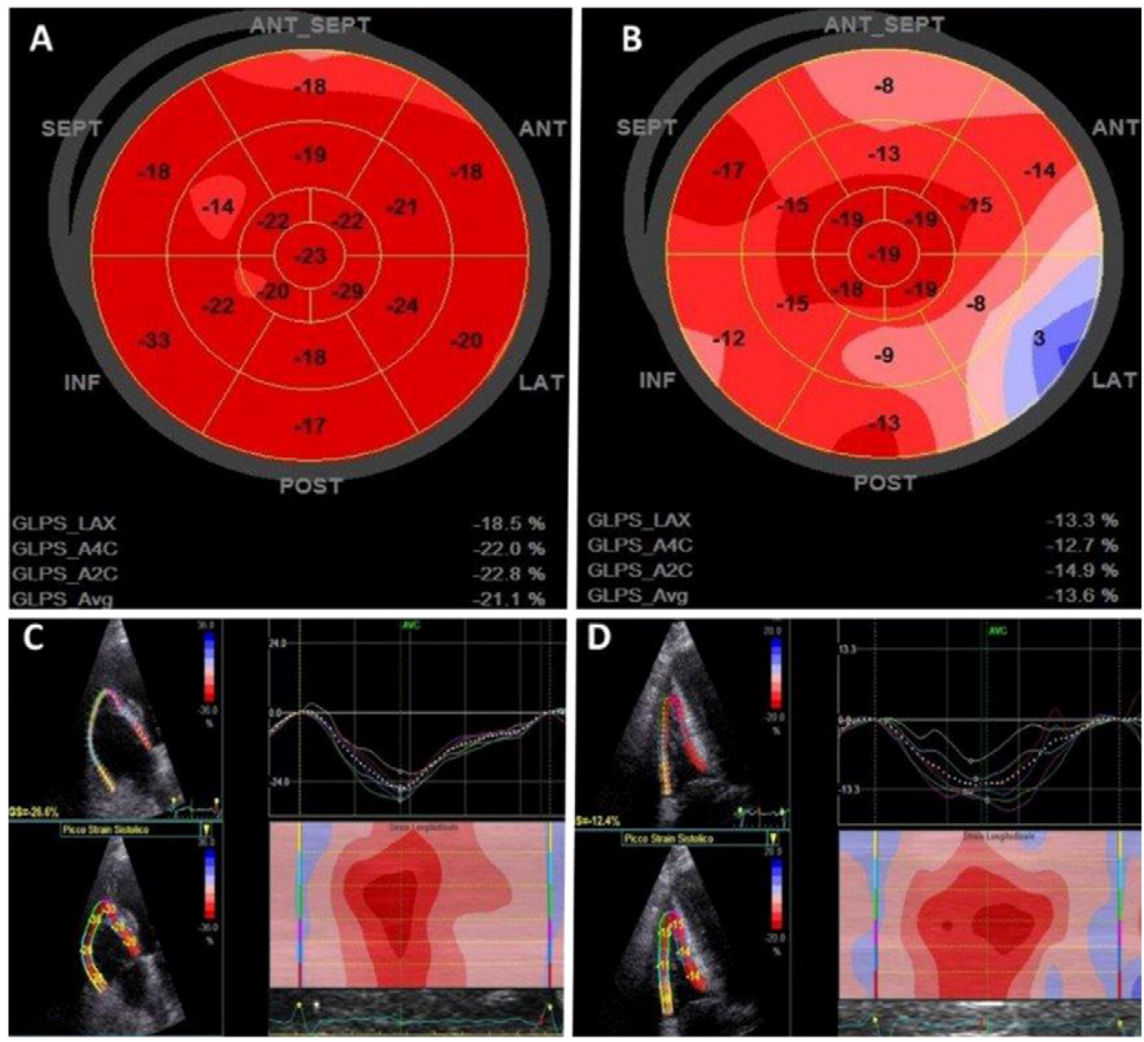

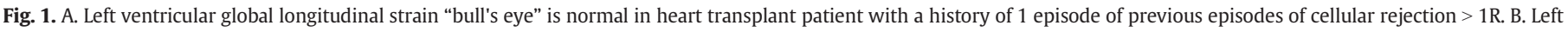

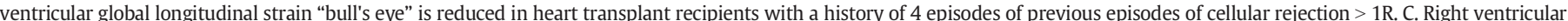

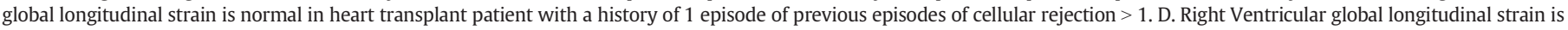
reduced in heart transplant recipients with a history of 4 episodes of previous episodes of cellular rejection $>1 \mathrm{R}$.

the ISHLT classification [3] and was associated with the number of PreCR $(\mathrm{OR}=1.8$; [95\% CI, 1.10-2.96], $\mathrm{p}=0.02)$. In patients with 3 or more episodes of PreCR we observed lower average values of LVGLS of $-11.9 \pm 1.06 \%$ compared with patients with no PreCR relapses $(-16.8 \pm 3.9 \%$, see Fig. 1). No correlation was found between number of PreCR and LVEF, tricuspid annular plane systolic excursion (TAPSE), pulsed wave tissue Doppler-derived tricuspid lateral annular systolic velocity $\left(\mathrm{S}^{\prime}\right)$ and RV fractional area change (FAC) (Table 2S). In HTx patients, there was no statistically significant association between LV- and RV-GLS and diabetes ( $p=0.14$ and $p=0.92$, respectively) or hypertension $(\mathrm{p}=0.11$ and $\mathrm{p}=0.14$, respectively). Considering LV radial strain at papillary muscle level, no association was found (Table 2). Free wall right ventricular longitudinal strain (FWRVLS) was related to RV-FAC

Table 1

Echocardiography data and strain analysis.

\begin{tabular}{lccc}
\hline Variable & \multicolumn{1}{l}{$\begin{array}{l}\text { HTx } \\
(\mathrm{n}=21)\end{array}$} & $\begin{array}{l}\text { Healthy controls } \\
(\mathrm{n}=21)\end{array}$ & p-Value \\
\hline Standard echocardiography & & & \\
$\quad$ EF, \% & $61 \pm 3,65$ & $62 \pm 2.5$ & 0.347 \\
$\quad$ TAPSE, mm & $12.9 \pm 32.4$ & $24.9 \pm 2.4$ & 0.000 \\
Wave S', m/sec & $0.06 \pm 0.01$ & $0.12 \pm 30.01$ & 0.000 \\
FAC, \% & $48 \pm 5$ & $52 \pm 6$ & 0.016 \\
Speckle tracking analysis & & & \\
LVRS, \% & $+34.36 \pm 2.9$ & $+49 \pm 17.3$ & 0.001 \\
LVGLS, \% & $-15.14 \pm 3.94$ & $-20 \pm 1.27$ & 0.0001 \\
RVGLS, \% & $-17.22 \pm 4.13$ & $-25.47 \pm 3.23$ & 0.0001 \\
FWRVLS, \% & $-21.54 \pm 4.26$ & $-29.5 \pm 4.8$ & 0.0001 \\
\hline
\end{tabular}

HTx: heart transplantation; EF: ejection fraction; TAPSE: tricuspid annular plane systolic excursion; FAC: fractional area change; LVRS: left ventricular systolic radial strain; LVGLS: left ventricular global longitudinal strain; RVGLS: right ventricular global longitudinal strain; FWRVLS: free wall right ventricular longitudinal strain.
( $p<0.001$ ) (Table 2 and Fig. 3S), although no correlation was found with TAPSE $(\mathrm{p}=0.46)$ and $\mathrm{S}^{\prime}(\mathrm{p}=0.39)$ (Table 2$)$.

\section{Discussion}

The main findings of the current investigation are as follows: 1) in patients referred to HTx, systolic LV-GLS and RV-GLS being abnormally reduced with a trend resembling distribution of worse than $1 \mathrm{R}$ rejections, were the most sensitive ECHO measurements to detect early, subclinical bi-ventricular impairment; 2) FWRVLS is the most accurate ECHO measure to assess RV performance in this population, superior to standard parameters such as TAPSE or $\mathrm{S}^{\prime}$ velocity that are possibly influenced by previous cardiac surgery; finally, 3) LVEF, being stable even in patients with several rejections, is not helpful in depicting early myocardial dysfunction in this population.

The sensitivity of longitudinal systolic strain in detecting myocardial impairment in patients undergone HTx, has been already reported [4]. The significant correlation between LVGLS and RVGLS and the association of these two parameters with multiple episodes PreCR highlights the critical role of deformation imaging in detecting early cardiac impairment: indeed, several observations in different cardiomyopathies have shown that myocardial contractile function is initially impaired just in the longitudinal direction [5].

Although our study was not aimed at quantitating specifically myocardial fibrosis, it is reasonable to hypothesize that LV impairment may be due to tissue fibrosis related to long-term effects of recidivant PreCR.

It is noteworthy that, while RV-GLS was consistent to LV-GLS and associated to the number episodes of PreCR, this was not true for FWRVLS: since RV-GLS includes strain of the right side of the infero-septal wall, that is also the preferred EMB location, it is therefore possible that effect 
Table 2

Correlation (Pearson correlation or Spearman's* rho) between different variables and strain analysis.

\begin{tabular}{|c|c|c|c|c|}
\hline Variable & LVGLS & LVRS & RVGLS & FWRVLS \\
\hline \multicolumn{5}{|l|}{ EMB } \\
\hline PreCR & $0.44 ; p=0.045$ & $-0.31 ; p=0.200$ & $0.49 ; \mathrm{p}=0.019^{*}$ & $0.23 ; p=0.284^{*}$ \\
\hline \multicolumn{5}{|c|}{ Standard echocardiography } \\
\hline $\mathrm{EF}, \%$ & $-0.56 ; p=0.006^{*}$ & $-0.06 ; p=0.785$ & $-0.20 ; p=0.355^{*}$ & $-0.14 ; p=0.496$ \\
\hline $\mathrm{E} / \mathrm{e}^{\prime}$ & $0.02 ; p=0.940$ & NA & NA & \\
\hline TAPSE, mm & NA & NA & $-0.11 ; \mathrm{p}=0.631^{*}$ & $-0.17 ; p=0.469$ \\
\hline Wave s', m/sec & NA & NA & $-0.20 ; p=0.390$ & $-0.20 ; p=0.390$ \\
\hline FAC, $\%$ & NA & NA & $-0.81 ; \mathrm{p}<0.001$ & $-0.81 ; \mathrm{p}<0.001$ \\
\hline \multicolumn{5}{|l|}{ Other variables } \\
\hline Ischemia time & $-0.32 ; \mathrm{p}=0.171^{*}$ & $0.19 ; p=0.477$ & $-0.06 ; p=0.772^{*}$ & $-0.05 ; \mathrm{p}=0.813^{*}$ \\
\hline Time after HTx & $-0.17 ; \mathrm{p}=0.452^{*}$ & $-0.06 ; p=0.813$ & $-0.03 ; p=0.863^{*}$ & $-0.07 ; p=0.733$ \\
\hline
\end{tabular}

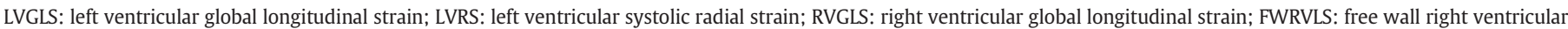

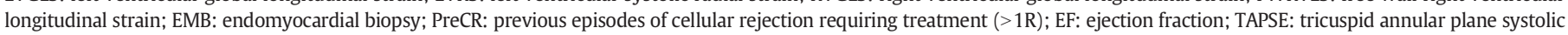
excursion; FAC: fractional area change; HTx: heart transplantation.

of worse than $1 \mathrm{R}$ rejections on RV-GLS was magnified by EMB-induced iatrogenic fibrosis. On the other hand, strain of the RV free wall, although reduced in HTx patients as compared to healthy subjects, was not related to the amount of myocardial fibrosis. Our explanation is that FWRVLS, being independent from EMB-induced fibrosis, is likely a more objective parameter of RV function in patients undergoing multiple EBMs. Moreover, the reduction of TAPSE and $S^{\prime}$ velocity in patients referred to cardiac surgery is well known and not related to a real RV impairment [6]. Therefore, the abnormally low TAPSE and $\mathrm{S}^{\prime}$ velocity observed in HTx patients could just be a spurious result, where strain measurements, and in particular FWRVLS are more objective parameters of RV performance [7]. Failure to improve LVGLS after transplantation is associated with a higher incidence of cardiac events and death as demonstrated by Eleid et al. [8]. These results were independent from biopsy detected acute cellular rejection, suggesting that two dimensional speckle tracking echocardiography is able to detect early and subtle alteration of LV systolic function, with significant prognostic implications, in spite of a normal LVEF and RV-FAC.

\section{Conclusions}

HTx recipients with normal LVEF, multiple episodes of PreCR worse than $1 \mathrm{R}$ increase risk of abnormal myocardial fibrosis, leading to subclinical myocardial dysfunction. Standard Echocardiography measures including TAPSE or tricuspid annulus $S^{\prime}$ velocity are not reliable in depicting such dysfunction, while RV-GLS is prone to EMBinduced iatrogenic fibrosis. LV-GLS by 2D Speckle Tracking is sensitive in depicting pre-clinical myocardial impairment in this population and should be routinely measured in all patients followed-up after HTx, specifically in those developing any grade of cellular rejection.

\section{Conflict of interest}

The authors declare no actual or potential competing financial interests.

\section{Acknowledgments}

The authors would like to thank the Department for the Treatment and Study of Cardiothoracic Diseases and Cardiothoracic Transplantation at IRCCS - ISMETT (Istituto Mediterraneo per i Trapianti e Terapie ad alta specializzazione), Palermo, Italy, for continual clinical and intellectual support.

\section{Appendix A. Supplementary data}

Supplementary data to this article can be found online at http://dx. doi.org/10.1016/j.ijcard.2016.02.025.

\section{References}

[1] L.P. Badano, M.H. Miglioranza, T. Edvardsen, et al., European Association Of Cardiovascular Imaging/Cardiovascular Imaging Department of the Brazilian Society of Cardiology recommendations for the use of cardiac imaging to assess and follow patients after heart transplantation, Eur. Heart J. Cardiovasc. Imaging 16 (2015) 919-948.

[2] T.S. Clemmensen, B.B. Løgstrup, H. Eiskjær, S.H. Poulsen, Changes in longitudinal myocardial deformation during acute cardiac rejection: the clinical role of two-dimensional speckle-tracking echocardiography, J. Am. Soc. Echocardiogr. 28 (2015) 330-339.

[3] S. Stewart, G.L. Winters, M.C. Fishbein, et al., Revision of the 1990 working formulation for the standardization of nomenclature in the diagnosis of heart rejection, J. Heart Lung Transplant. 24 (2005) 1710-1720.

[4] P. Pichler, T. Binder, P. Höfer, et al., Two-dimensional speckle tracking echocardiography in heart transplant patients: three-year follow-up of deformation parameters and ejection fraction derived from transthoracic echocardiography, Eur. Heart J. Cardiovasc. Imaging 13 (2012) 181-186.

[5] D. Bellavia, P. Pellikka, A. Dispenzieri, et al., Comparison of right ventricular longitudinal strain imaging, tricuspid annular plane systolic excursion, and cardiac biomarkers for early diagnosis of cardiac involvement and risk stratification in primary systematic (AL) amyloidosis: a 5-year cohort study, Eur. Heart J. Cardiovasc. Imaging 13 (2012) 680-689.

[6] G. Tamborini, M. Muratori, D. Brusoni, et al., Is right ventricular systolic function reduced after cardiac surgery? A two- and three-dimensional echocardiographic study, Eur. J. Echocardiogr. 10 (2009) 630-634.

[7] M.C. Todaro, G. Romano, S. Carerj, F. Clemenza, M. Pilato, B. Khandheria, Right ventricular free wall strain: a predictor of successful left ventricular assist device implantation, Tex. Heart Inst. J. 42 (2015) 87-89.

[8] M.F. Eleid, G. Caracciolo, E.J. Cho, et al., Natural history of left ventricular mechanics in transplanted hearts: relationships with clinical variables and genetic expression profiles of allograft rejection, JACC Cardiovasc. Imaging 3 (2010) 989-1000. 